\title{
Longitudinal behavioral and fMRI-based assessment of inhibitory control in heroin addicts on methadone maintenance treatment
}

\author{
JIAN-JUN YE*, WEI LI*, DONG-SHENG ZHANG, QIANG LI, JIA ZHU, JIA-JIE CHEN, YONG-BIN LI, \\ XUE-JIAO YAN, JIE-RONG LIU, XUAN WEI, YA-RONG WANG and WEI WANG
}

Department of Radiology, Tangdu Hospital, The Fourth Military Medical University, Xi'an, Shaanxi 710038, P.R. China

Received October 8, 2016; Accepted June 5, 2017

DOI: $10.3892 / \mathrm{etm} .2018 .6571$

\begin{abstract}
The objective of the present study was to determine whether methadone maintenance treatment (MMT) in heroin-dependent patients affects inhibitory control, whether any MMT-induced changes correlate with methadone dose and MMT duration, and whether these changes depend on the psychological characteristics of patients, such as depression, anxiety and impulsivity. Response inhibition in the GO/NO-GO test was combined with functional magnetic resonance imaging (fMRI) scanning data to examine whether MMT affects inhibitory control in 21 heroin-addicted patients who had already undergone at least three months of MMT. Patients were evaluated one year prior to and after the MMT period. Participants exhibited no difference in their GO/NO-GO reaction time and accuracy rate, or in their false alarm rate under NO-GO conditions. However, increased activation was detected in numerous brain regions in their 12-month fMRI scans, although these were not in the frontal-striatal loop. Increased fMRI activation in the left precentral gyrus and superior temporal gyrus were negatively correlated with the daily methadone dose and total methadone dose during the one-year study period. In conclusion, these results suggested that MMT over one year does not significantly change the behavioral indicators of inhibitory control function in heroin-dependent patients. The increase in activation leads to the hypothesis that MMT over one year may increase cognitive inhibitory control, which may be the result of the combined negative effect of methadone and the positive effect of functional recovery after withdrawal of heroin.
\end{abstract}

Correspondence to: Dr Wei Wang, Department of Radiology, Tangdu Hospital, The Fourth Military Medical University, 569 Xinsi Road, Baqiao, Xi'an, Shaanxi 710038, P.R. China

E-mail:ww0161doc@163.com

*Contributed equally

Key words: fMRI, inhibitory control, heroin addicts, methadone maintenance treatment

\section{Introduction}

Heroin addiction is a global problem with widespread social and public health implications. Methadone maintenance treatment (MMT) has been widely used to treat patients addicted to heroin. However, it remains controversial whether methadone is effective or not.

Behavioral studies (1-4) have demonstrated that patients dependent on heroin or other opioids have a significantly lower inhibitory control function than normal volunteers. Animal experiments in mice have revealed that methadone treatment $(2.5-10 \mathrm{mg} / \mathrm{kg})$ once daily for three weeks with repeated withdrawals on Saturdays and Sundays has a negative impact on cognitive function, regardless of whether methadone is detectable in brain tissues (5). In addition, neural apoptotic damage caused by chronic methadone administration occurs in mouse brain white matter (6) and correlates with memory dysfunction and depression in former heroin users receiving MMT (7).

Certain studies provided behavioral evidence that MMT improves inhibitory control. Using a reaction time test based on a stop-signal task (SST), one study (8) revealed that prior to MMT, the reaction time in opioid-dependent individuals was significantly longer than that in healthy controls. However, this difference disappeared after these dependent individuals had been opioid-abstinent for two months with MMT. Another study used the digit symbol substitution task and continuous performance task to assess psychomotor speed, and selective attention/impulsivity assessment revealed that methadone-maintained individuals performed worse than controls after $<1$ year of MMT. However, their performance approached that of controls after longer treatment (1). A study that used the Stroop Color-Word test to examine verbal learning, visuospatial memory and psychomotor speed suggested that opioid-dependent subjects exhibit significant improvement in cognitive function after two months of MMT (9).

The disagreement between studies regarding whether MMT affects inhibitory control function likely reflects the inadequate consideration of numerous factors that may affect it, including social support, personality and cognitive as well as neural factors (10). For instance, depression is ubiquitous among patients receiving MMT, although different studies report a wide variety of prevalence rates (11) and depressive disorders are associated with interference control (12). A voxel-based morphometric study revealed that MMT patients 
have structural deficits in the emotion control circuit and cerebellum, which are associated with depression, anxiety and cognitive dysfunction (13). Using diffusion tensor imaging, MMT patients were found to have more memory and emotional deficits than healthy subjects (7). Furthermore, significant differences were also found in the white matter content of the reward circuit and in depression- and memory-associated regions. Individuals with high impulsivity and low inhibitory control are more likely to become dependent on drugs (14). In addition, few event-associated functional magnetic resonance imaging (fMRI) studies have directly examined how therapy curbs the addictive thoughts and behaviors of patients. Another noteworthy phenomenon is that most former studies on MMT have been cross-sectional, preventing investigators from adequately controlling for possible effects of previous addictive drug use. Understanding whether and how MMT affects inhibitory control function is essential for improving efficacy, which is particularly important, as therapy is associated with a relatively high rate of relapse (15).

In order to address these gaps in the literature, the present study combined behavioral experiments with fMRI and examined whether MMT alters particular neuronal circuits associated with inhibitory control. This technique has been demonstrated to provide sufficient temporal resolution to capture changes in inhibitory control $(16,17)$. Previous fMRI studies have indicated that significant activation of inhibitory control primarily occurs in the bilateral medial prefrontal cortex, anterior cingulate cortex and inferior frontal gyrus (IFG) in healthy controls (18-20). Experiments using fMRI have already revealed that fewer brain regions and smaller overall brain areas are activated under conditions of inhibitory control in heroin-dependent individuals compared with those in healthy controls (18).

The objective of the present study was to determine whether MMT in heroin-dependent patients affects inhibitory control, whether any MMT-induced changes correlate with the methadone dose and MMT duration, and whether these changes depend on the psychological characteristics of patients such as depression, anxiety and impulsivity.

\section{Materials and methods}

Design. In the present study, performance in the classical GO/NO-GO task at baseline and after one year of MMT was assessed. Similar to SST, this is often used to assess the inhibitory control of the motor response (21-24). These tests were complemented with several standard behavioral assessments of inhibitory function, including the Hamilton anxiety scale (HAMA) (25), the Beck Depression Inventory-II (BDI-II) (26), the Protracted Withdrawal Symptoms Scale (PWSS) (27) and the Barratt Impulsivity Scale 11 (BIS-11) (28).

Participants. The study protocol was approved by the Tangdu Hospital Review Board (the Fourth Military Medical University, Xi'an, China). MMT subjects were recruited from outpatients receiving standard treatment at one of the Baqiao methadone substitution treatment centers in Xi'an (China). All participants provided written informed consent prior to entering into the study. These patients underwent behavioral tests and fMRI scans between October and December 2012, and these were taken again one year later.
Inclusion criteria were as follows: i) Right-handedness, normal vision and an age of 18-50 years; ii) heroin dependence according to the Diagnostic and Statistical Manual of Mental Disorders, Fourth Edition, Text Revision (DSM-IV-TR) criteria (29); iii) a score of $>90$ points on Raven's intelligence test, with no clinically significant deficits in intelligence or language communication; iv) a history of heroin use followed by complete detoxification; v) intake of a stable dose of methadone for at least three months prior to enrollment into the study.

Subjects were excluded from the study if they presented any of the following: i) history of using cocaine or other drugs of abuse besides heroin; ii) current alcohol intake of $>15$ drinks (210 g alcohol; $1.5 \mathrm{oz}$ of liquor or $12 \mathrm{oz}$ of beer) per week; iii) cerebral organic disease; iv) positivity for human immunodeficiency virus; v) neurological signs and/or history of neurological disease in the patient or first-degree relatives; vi) decreased hepatic, renal, or cardiac function, and history of long-term use of associated drugs; vii) claustrophobia or other MRI contraindications; viii) a history of cardiovascular or endocrine disease; ix) a current medical illness or recent medicine use; $\mathrm{x}$ ) fMRI data showing $>1.5 \mathrm{~mm}$ of displacement and/or $>1.5^{\circ}$ rotation in any of the axes during any of the task repetitions; or xi) a combined accuracy rate $<90 \%$ on the GO and NO-GO tasks and an error rate of $>50 \%$ on the NO-GO task.

Procedure. The test took $50 \mathrm{~min}$ to complete. In the first step, clinico-demographic and psychological scale data were collected (Tables I and II). In the second step, fMRI scans and the $\mathrm{GO} / \mathrm{NO}$-go task were simultaneously performed. Once fMRI data were acquired, participants were paid and verbally debriefed.

Assessments. Clinico-demographic data collection. Clinico-demographic characteristics of patients, as well as their history of heroin use and methadone treatment prior to the start of the one-year MMT period, were recorded. Patients were also assessed at baseline for severity of psychological characteristics using HAMA, BDI, PWSS and BIS-11. Patients were assessed again one year later using the same instruments.

Relapse detection. To detect relapse events, participants were interviewed monthly and their urine was tested for heroin.

GO/NO-GO test. The GO/NO-GO tasks were performed in two runs $(18,24,30)$, which consisted of $316 \mathrm{GO}$ blocks and 20 NO-GO blocks, which lasted for $346 \mathrm{sec}$. The tasks were performed during each testing session. Participants lay quietly in the MRI scanner during testing. They were presented with a text description of the task for $10 \mathrm{sec}$ prior to commencement. During the GO trials, participants were presented with the letters ' $\mathrm{X}$ ' or ' $\mathrm{Y}$ ' alternately. Letters appeared in white on a black background at a resolution of 640x640; the letter appeared for $900 \mathrm{msec}$, followed by a black screen that lasted for $100 \mathrm{msec}$ (stimulus interval). Participants were instructed to press button ' 1 ' on their keypad as soon as they saw the letter ' $\mathrm{X}$ ', and press button ' 2 ' when they saw the letter ' $\mathrm{Y}$ '. If the alternate rule was broken (as in the NO-GO trials), they were instructed not to press any button. NO-GO trials 
Table I. Demographic characteristics of heroin-dependent Han Chinese patients undergoing methadone maintenance treatment.

\begin{tabular}{lcc}
\hline Characteristic & Value & Range \\
\hline Age, years & $35.8 \pm 8.0$ & $22-48$ \\
Education, years & $9.3 \pm 2.1$ & $6-12$ \\
Cigarette use & & $2-30$ \\
Overall duration, years & $17.2 \pm 8.2$ & $5-45$ \\
Daily amount, $\mathrm{n}$ & $19.0 \pm 8.2$ & $3,650-306,600$ \\
Lifetime amount, $\mathrm{n}$ & $123,839.2 \pm 77,947.6$ & $0.5-19$ \\
Heroin use & & $0.1-2.0$ \\
Overall duration, years & $7.3 \pm 6.5$ & $78-6,852$ \\
Daily dose, g/day & $0.5 \pm 0.4$ & $0.3-4.7$ \\
Lifetime dose, $g$ & $1,105.2 \pm 1,525.1$ & $12-80$ \\
Methadone use & & $9.480-162,425$ \\
Overall duration, years & $42.2 \pm 1.4$ & $4,320-28,440$ \\
Daily dose, mg & $50,994.7 \pm 35,460.4$ & $15,188.6 \pm 6,000.9$ \\
Lifetime dose, mg & & \\
Total dose during the 1-year study period, $m g$ &
\end{tabular}

Table II. Personality and psychological characteristics of heroin-dependent Han Chinese patients prior to and after 1-year methadone maintenance treatment.

\begin{tabular}{|c|c|c|c|c|}
\hline Survey & Baseline value & Value after 1-year therapy & $\mathrm{t}$-value & P-value \\
\hline BDI & $9.3 \pm 8.1$ & $7.7 \pm 8.3$ & 1.151 & 0.263 \\
\hline HAMA & $6.4 \pm 7.6$ & $7.9 \pm 6.3$ & -1.104 & 0.283 \\
\hline PWSS & $12.3 \pm 14.4$ & $9.8 \pm 11.0$ & 0.826 & 0.419 \\
\hline \multicolumn{5}{|l|}{ BIS } \\
\hline Total & $62.1 \pm 9.9$ & $62.6 \pm 7.1$ & -0.195 & 0.847 \\
\hline AI & $14.0 \pm 3.0$ & $14.3 \pm 2.8$ & -0.255 & 0.801 \\
\hline MI & $28.9 \pm 3.5$ & $19.4 \pm 3.3$ & 0.850 & 0.406 \\
\hline NPI & $27.9 \pm 5.5$ & $28.9 \pm 3.5$ & -0.708 & 0.487 \\
\hline
\end{tabular}

Differences between time-points were assessed for significance using the paired sample t-test. BDI, Beck Depression Inventory-II; HAMA, Hamilton Anxiety scale; PWSS, Protracted Withdrawal Symptoms Scale; BIS, Barratt Impulsivity Scale; AI, attention impulsivity; MI, motor impulsivity; NPI, non-planned impulsivity.

were pseudo-randomly interspersed throughout the GO trials. Performance measures on the GO/NO-GO task, including reaction time, accuracy rate and error rate, were collected and analyzed using E-prime version 2.0 (Psychology Software Tools, Pittsburgh, PA, USA).

Participants were instructed to stop taking methadone $20 \mathrm{~h}$ prior to the GO/NO-GO task, which was performed between 8:00 a.m. and 12:00 p.m.

MRI data acquisition. fMRI data were acquired from participants during the performance of the GO/NO-GO tasks. All imaging data were acquired on a 3.0T MRI scanner (GE Signa Excite HD; GE Healthcare, Little Chalfont, UK) equipped with an 8-channel head coil. After localizer and conventional anatomical scans, GO/NO-GO blood oxygenation level-dependent (BOLD) responses were measured as a function of time using a T2*-weighted, single-shot, echo-planar imaging sequence to acquire $\mathrm{T} 2 *$-weighted image volumes with approximate AC-PC alignment [repetition time (TR), 2,000 msec; echo time (TE), $30 \mathrm{msec}$, flip angle, $90^{\circ}$ ]. Each brain volume consisted of 32 transverse slices with the following characteristics: Matrix, 64 tri; field of view (FOV), 256x256 $\mathrm{mm}^{2}$; thickness, $4 \mathrm{~mm}$; no gap; spatial resolution, $4 \times 4 \times 4 \mathrm{~mm}^{3}$. Each patient performed two GO/NO-GO task run, 168 echoplanar volumes were collected in each run, therefore a total of 336 volumes were collected for each patient. A high-resolution T1-weighted structural image was acquired using a 166-slice, high-resolution, fast-spoiled, gradient-echo 3-dimensional T1-weighted imaging sequence with the following characteristics: TR, $7.8 \mathrm{msec}$; TE, $3.0 \mathrm{msec}$;

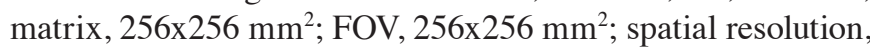
$1 \times 1 \times 1 \mathrm{~mm}^{3}$. 
MRI data processing. Structural data were first checked for abnormalities independently by two experienced radiologists (Yarong Wang and Wei Wang). Discrepancies were resolved through consultation. Data were then analyzed using Statistical Parametric Mapping 8 software (www.fil.ion.ucl.ac.uk/spm). A 6-parameter rigid-body transformation involving three rotations and three translations was used to register images and correct for head motion. Re-aligned datasets were spatially normalized to the standard stereotactic space of the Montreal Neurological Institute (MNI) using a 12-parameter affine transformation and a voxel size of $3 \times 3 \times 3 \mathrm{~mm}^{3}$. Subjects that had $>1.5 \mathrm{~mm}$ of head translation or $>1.5^{\circ}$ head rotation were excluded from the analysis. Data were spatially smoothed using an 8-mm full-width-half-maximum Gaussian kernel.

The smoothed data were then analyzed using a General Linear Model (17). Individual contrast images captured under NO-GO conditions were compared with individual images taken under the GO condition. In the second-level analysis, these images were compared between the two groups using a paired sample t-test. Subtraction results were presented by overlaying the statistical maps onto the standardized MNI anatomical image, with a threshold of $\mathrm{P}<0.05$ (AlphaSim-corrected) and a cluster size of $\geq 12$ voxels. For further analysis, regions of interest (ROIs) were defined by positioning spheres with a 2-mm radius at the local maxima in the statistical map for each cluster.

Statistical analysis. Statistical analysis was performed using SPSS 16.0 (SPSS, Inc, Chicago, IL, USA), and the threshold of significance was $\mathrm{P}<0.05$. Normally distributed data were expressed as the mean \pm standard deviation and assessed for significant differences between baseline and after one year of MMT using the paired sample t-test. Bivariate Pearson correlation analysis was used to examine possible associations between the percentual change in ROI signal intensity over the 1-year MMT period, as well as the clinico-demographic characteristics and behavioral indicators of the inhibitory control.

\section{Results}

Participants. Recruitment information was distributed to 139 heroin addicts undergoing regular MMT at Baqiao methadone substitution treatment centers in Xi'an (China). A total of 78 subjects were initially recruited, of which 73 were males. However, 37 subjects failed to show up for baseline testing. Among the 41 subjects who performed baseline testing, 15 were lost to follow-up. The remaining 26 subjects underwent testing at baseline as well as one year later. However, five subjects had to be excluded due to excessive motion during fMRI scanning or because their performance on the GO/NO-GO task fell within the exclusion criteria. In the end, the data of $21 \mathrm{Han}$ Chinese men (mean age, 35.8 years; range, 22-48 years) were included in the final analysis (Table I).

During the one-year study period, monthly urine testing detected 12 individuals who relapsed for an average of three times. The average relapse dose was $0.4 \mathrm{~g}$ based on follow-up interviews.

Depression and anxiety characteristics. The BDI, HAMA, PWSS and BIS-11 scores of the participants prior to and after the 1-year MMT period were similar (Table II).
Inhibitory control behavior in the GO/NO-GO task. Participants revealed no differences between baseline and at 12 months in terms of reaction time, accuracy rate under GO conditions, or false alarm rate under NO-GO conditions $(\mathrm{P}<0.05$; Table III).

Inhibitory control-associated neural activity during the GO/NO-GO task. Baseline fMRI scans taken prior to the one-year MMT revealed numerous regions of increased activation during the GO/NO-GO task (Fig. 1A). These included the bilateral occipital lobe (cuneus and superior occipital lobe), bilateral parietal lobe (superior parietal lobe, inferior parietal lobule and precuneus), bilateral frontal lobule (IFG, superior frontal gyrus, middle frontal gyrus and precentral gyrus), limbic system (anterior cingulated cortex, insula, back of the hippocampus and left parahippocampal gyrus), thalamencephalon (caudate nucleus, lentiform nucleus, claustrum and red nucleus), and cerebellum. Several regions of decreased activation were also observed. These included left cuneus, bilateral paracentral lobule, left precentral gyrus and left superior temporal gyrus.

On repeated fMRI scanning using the same protocol after one year of MMT, the levels of activation were found to be similar to those at baseline, although the overall area of activation was greater (Fig. 1B). By contrast, this decreased activation was observed in only two areas: Left postcentral gyrus and right precuneus.

Differential analysis revealed that the following regions had significantly higher activation levels at one year than at baseline (AlphaSim-corrected $\mathrm{P}<0.005$ ): Bilateral superior temporal gyrus, precentral gyrus and insula; left postcentral gyrus, cuneus, supramarginal gyrus, inferior parietal lobule and lingual; right IFG and middle cingulate gyrus (Fig. 2; Table IV). No brain areas were found to exhibit decreased activation after the one-year MMT.

Correlations. Changes in fMRI signal intensities between baseline and at one year were found to significantly correlate with one of the clinico-demographic characteristics (Fig. 3). These were negatively correlated with the total methadone dose during the one-year study period and with the methadone dose per day in the left precentral gyrus $(\mathrm{P}=0.013, \mathrm{r}=-0.534)$ and in the left superior temporal gyrus $(\mathrm{P}=0.001, \mathrm{r}=-0.661)$.

At the same time, intensity changes in brain activation did not display any significant associations with the duration of heroin use, lifetime heroin dose or inhibitory control behavior (data not shown).

\section{Discussion}

The present study aimed to clarify the controversial question of whether MMT significantly affects inhibitory control function in heroin-dependent patients, which was assessed in the present study using a classical GO/NO-GO task. This behavioral task was coupled with fMRI to capture dynamic changes in neural circuit activation, which are potentially associated with inhibitory control. In order to avoid certain limitations of previous studies, the present study performed behavioral and fMRI measurements at baseline and after one year of MMT. This did not compare patients with healthy participants, thereby helping to isolate the effects of methadone from those of heroin. 
Table III. Characteristics of inhibitory control behavior of patients during execution of the GO/NO-GO task at baseline and after 1 year of methadone maintenance treatment.

\begin{tabular}{lccrr}
\hline Items & Baseline value & Value after 1-year therapy & t-value & P-value \\
\hline Average reaction time, msec & $407.33 \pm 71.99$ & $400.30 \pm 77.65$ & 0.337 & 0.710 \\
Average accuracy rate on GO, \% & $0.98 \pm 0.03$ & $0.98 \pm 0.03$ & -0.296 & 0.770 \\
False alarm rate on NO-GO, \% & $0.32 \pm 0.12$ & $0.28 \pm 0.14$ & 0.924 & 0.366 \\
\hline
\end{tabular}

Differences between time-points were assessed for significance using the paired sample t-test.

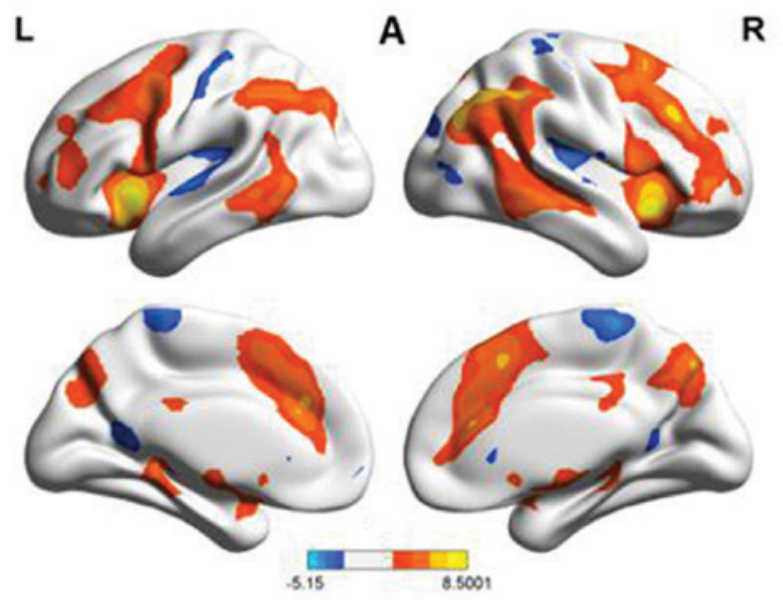

L

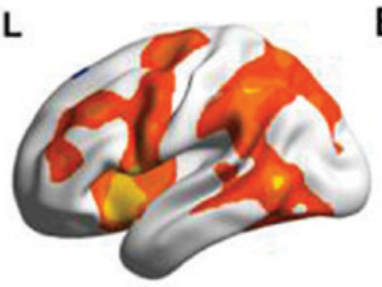

B
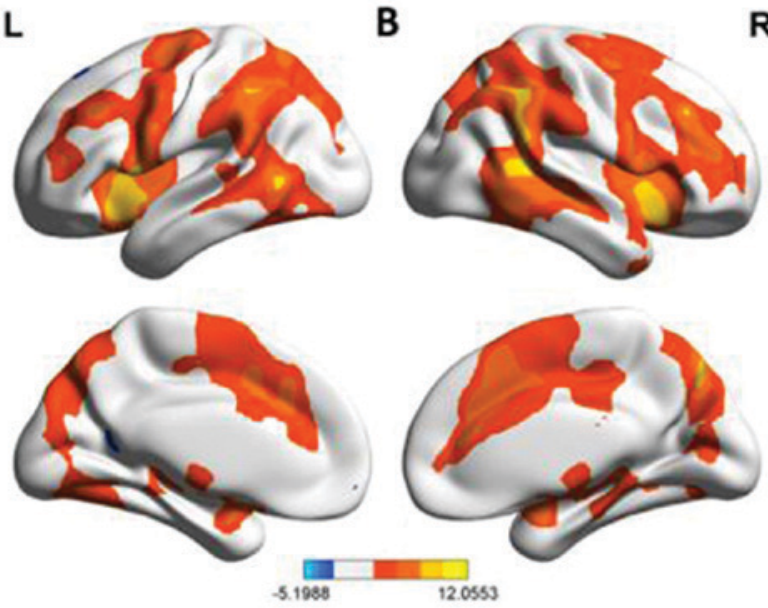

Figure 1. Average activation in the brains of 21 heroin-dependent Han Chinese men (A) at baseline and (B) after 1 year of methadone maintenance treatment. Axial t-maps of brain activation (AlphaSim-corrected $\mathrm{P}<0.005$ ) were superimposed on a standard Statistical Parametric Mapping anatomical template brain according to neurological conventions. The $\mathrm{z}$ coordinate in the Talairach space is presented for each slice. L, left; R, right.

The present results suggested that one year of MMT did not significantly change the personality inventory scores or behavioral indicators of the inhibitory control function in heroin-dependent patients. This is inconsistent with findings of previous studies $(3,8-10)$. This apparent discrepancy may reflect the fact that these previous studies were cross-sectional, whereas the present study was longitudinal, which provided a potentially more reliable assessment of the effects of MMT. In addition, previous studies compared their patients with healthy controls, which meant that the outcomes may have been attributed to the effects of heroin use, MMT or both. The present study included only patients with a history of heroin use, who had been undergoing MMT. Hence, the key difference between measurements at baseline and at one year may only be MMT.

As far as we are aware, the effects of methadone on inhibitory control in humans have not been previously reported, although studies in animals suggested that the drug reduces inhibitory control (4). The observation of no significant change in inhibitory control behavior in the GO/NO-GO task prior to or after one year of MMT may mean that MMT actually augments inhibitory control in the long term. If MMT initially induced such a decrease in the heroin-dependent subjects of the present study, this would imply that there was a prompt recovery to offset the damage. The event-associated fMRI results further confirmed this hypothesis.

The fMRI scanning results of the present study demonstrated greater activation in regions involved in inhibitory control function after one year of MMT than at baseline. This is consistent with the idea that one year of MMT increased inhibitory control processing at the level of neural circuits, which may reflect the increase in inhibitory control ability to a certain extent. However, methadone is an opioid, and numerous studies on the effects of MMT have reported that it may decrease certain cognitive functions in the long term (1-4). These two inconsistent findings may be explained by dual functions, the negative methadone effect and the recovery of function after withdrawal of heroin. Functional recovery after withdrawal of opioids has also been confirmed by numerous studies (31-34). This hypothesis was also supported by the correlation analysis results between the change in signal intensity of brain activation and the methadone dose found in the present study.

Increased BOLD intensity in the left precentral gyrus and superior temporal gyrus was negatively correlated with the methadone dose per day (mg/day) and the total methadone dose during the one-year study period (mg), suggesting that methadone negatively affected the activity in these brain regions. The increase in signal may be explained by the functional recovery after the withdrawal of heroin. In addition, the negative effects of methadone appear to be outweighed by overall functional recovery. Studies have demonstrated that heroin abstainers gradually recover their inhibitory control function with increasing abstinence time during MMT $(1,8)$. Consistent with these behavioral studies, an fMRI study revealed that certain brain regions linked to inhibitory control gradually became active with increasing 
Table IV. Clusters of significant differences in activation detected by functional magnetic resonance imaging in patients during execution of the GO/NO-GO task at baseline and after 1 year of methadone maintenance treatment (AlphaSim-corrected $\mathrm{P}<0.005$ ).

\begin{tabular}{|c|c|c|c|c|c|c|c|c|}
\hline Cluster & $\mathrm{L} / \mathrm{R}$ & Brain region & $\mathrm{x}$ & $\mathrm{y}$ & $\mathrm{z}$ & Voxel & $\mathrm{BA}$ & t-value ${ }^{a}$ \\
\hline \multirow[t]{2}{*}{1} & $\mathrm{~L}$ & Superior temporal gyrus & -60 & 4 & 2 & 165 & $22,41,42$ & 4.89 \\
\hline & $\mathrm{L}$ & Insula & -44 & -6 & 6 & 34 & 13 & 3.68 \\
\hline \multirow[t]{2}{*}{2} & $\mathrm{~L}$ & Lingual & -22 & -72 & -8 & 103 & 18,19 & 5.24 \\
\hline & $\mathrm{L}$ & Cuneus & -19 & -90 & 34 & 30 & 19 & 4.13 \\
\hline \multirow[t]{3}{*}{3} & $\mathrm{~L}$ & Postcentral gyrus & -64 & -26 & 20 & 45 & 40 & 4.08 \\
\hline & $\mathrm{L}$ & Inferior parietal lobule & -60 & -42 & 28 & 28 & 40 & 3.94 \\
\hline & $\mathrm{L}$ & Supramarginal gyrus & -60 & -44 & 30 & 17 & 40 & 3.98 \\
\hline \multirow[t]{3}{*}{4} & $\mathrm{R}$ & Superior temporal gyrus & 54 & 6 & 2 & 45 & 22 & 4.22 \\
\hline & $\mathrm{R}$ & Inferior frontal gyrus & 60 & 6 & 30 & 21 & 48 & 4.04 \\
\hline & $\mathrm{R}$ & Insula & 40 & -20 & 12 & 16 & 13 & 3.49 \\
\hline 5 & $\mathrm{~L}$ & Precentral gyrus & -58 & 0 & 7 & 40 & 6 & 3.98 \\
\hline 6 & $\mathrm{R}$ & Precentral gyrus & 62 & 2 & 29 & 48 & 4,6 & 4.21 \\
\hline 7 & $\mathrm{R}$ & Middle cingulate gyrus & 4 & -2 & 45 & 20 & 24 & 3.62 \\
\hline
\end{tabular}

${ }^{\mathrm{a}}$ From the paired t-test performed on measurements at the peak voxel within a cluster, i.e., the voxel showing the greatest statistical difference from the other voxels in the same cluster. $x, y, z$, coordinates according to the Montreal Neurological Institute; BA, Brodmann area; L/R, left/right cerebrum.
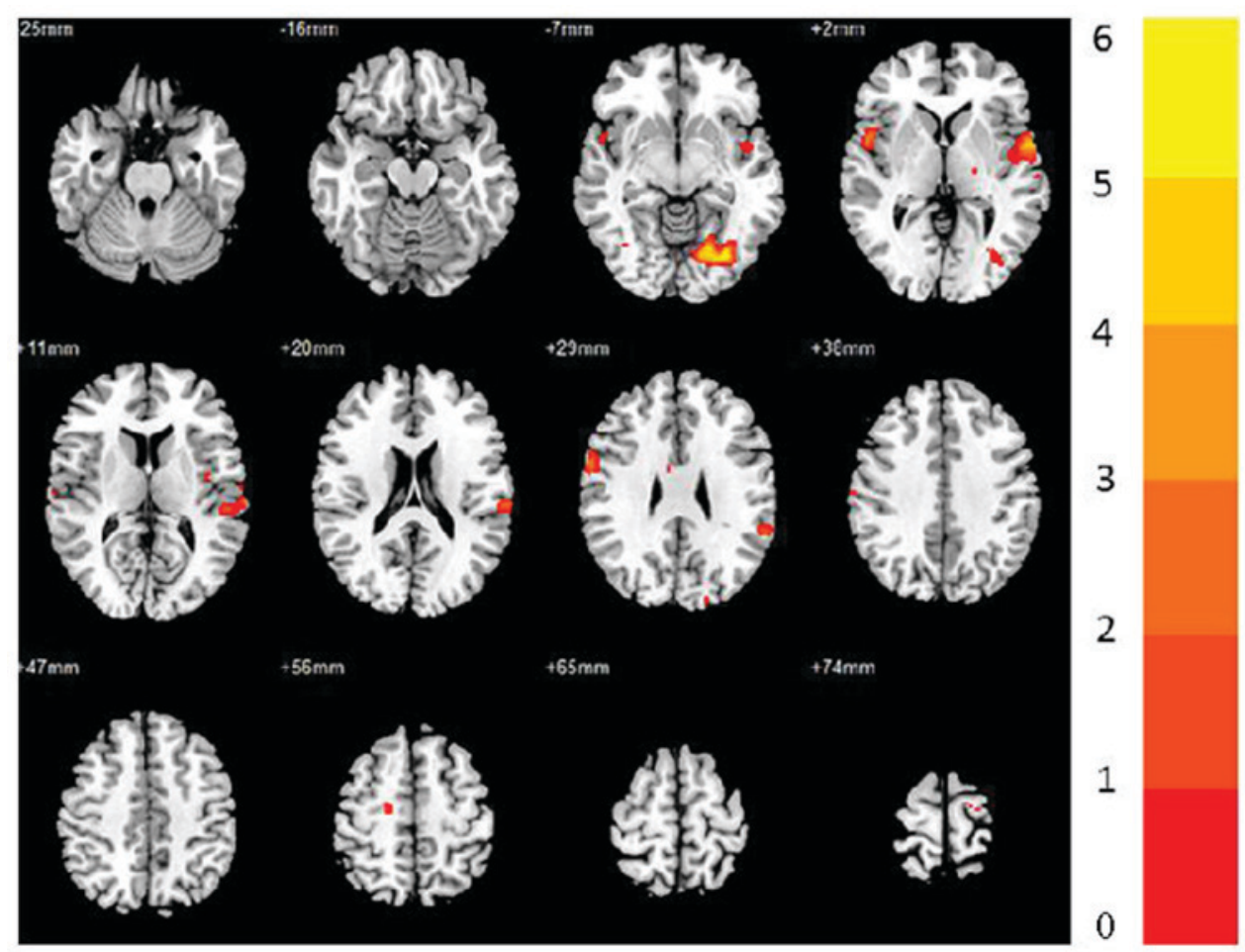

Figure 2. Maps of regions with differences in brain activation between baseline and after 1 year of methadone maintenance treatment. The 0-6 scale indicates the t-value of the activation intensity; the larger the t-value, the more significant the statistical difference. The bilateral superior temporal gyrus, precentral gyrus and insula, left postcentral gyrus, cuneus, supramarginal gyrus, inferior parietal lobule and lingual, right inferior frontal gyrus and middle cingulate gyrus have significantly higher activation levels at one year than at baseline (paired sample t-test, significant difference AlphaSim-corrected, $\mathrm{P}<0.005$ ).

abstinence time (31). The recovery of inhibitory control in heroin-abstinent individuals independent of MMT may be assessed by including an appropriate control arm in future studies. However, whether this would comply with international ethical standards remains controversial.
It was surprising that one-year MMT increased the activation of brain regions associated with inhibitory control without affecting inhibitory control behavior in the GO/NO-GO task. A possible explanation may be that one year of MMT exerts effects on brain regions associated with inhibitory 

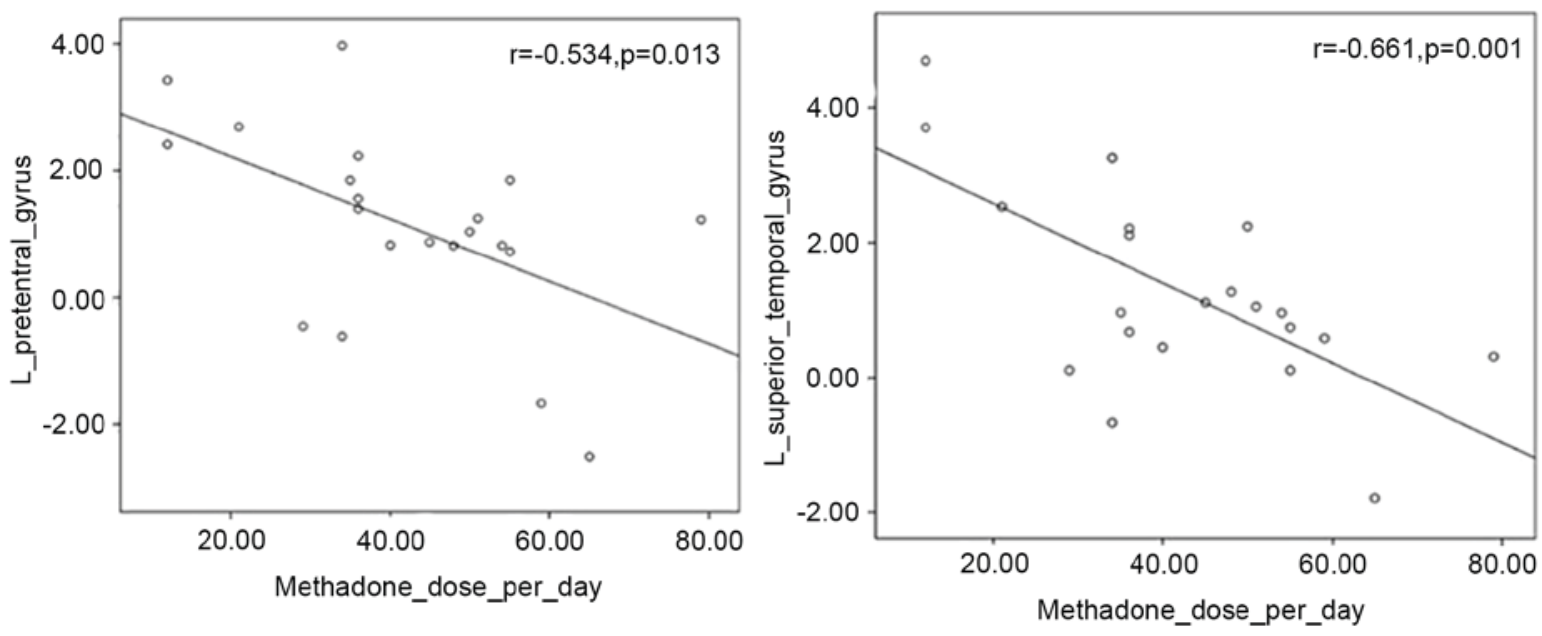

Figure 3. Correlations between the change in intensity of gray matter abnormalities and methadone dose per day.

control earlier than on inhibitory control behavior. In 1995 , Harnishfeger (35) proposed that inhibition occurs in two stages: Cognitive inhibition and behavioral inhibition; and this distinction continues to drive the field (36). Although two contrasting loop theories have been proposed to explain inhibitory control $(37,38)$, each of the two viewpoints that inhibitory control is a top-down process and moves from the back to the front of the brain have been confirmed (39). The interaction of the supplementary motor area with the subcortical brain and brain stem nuclei then completes the inhibitory control process (39). The forehead-striatal loop, which participates in motion control and response inhibition, is of particular importance (40-44). The GO/NO-GO task is expected to trigger cognitive as well as behavioral inhibition. All areas that display higher activation after MMT than at baseline are associated with the cognitive processes, including those occurring in the superior frontal gyrus, which is responsible for receiving and processing information $(20,21,30,40,45,46)$. However, no significant activation changes were observed in the forehead-striatal loop, particularly in the basal ganglia domain $(47,48)$. This may explain why MMT patients demonstrated no significant improvement in the behavioral test of inhibitory control. The possible interpretation of these findings is that MMT, for as short as one year, triggers improvement in the cognitive inhibitory control processes. However, this time may be too short to strengthen behavioral pathways. Future studies should examine the two types of inhibitory control in greater detail over a longer MMT.

The present study lays the groundwork for follow-up studies to flesh out the effects of MMT at neural and behavioral levels, but its results should be interpreted with caution. One major limitation is the small sample size and heterogeneity in patient characteristics, which may explain why the present study failed to observe correlations of brain activity with either the duration of heroin use, which ranged widely from 0.5 to 19 years, or the lifetime heroin dose, which ranged from 78 to $6,852 \mathrm{~g}$. Low statistical power may also explain why the present study failed to observe significant changes in behavioral inhibitory controls. All study participants were male, highlighting the requirement to perform similar studies in females. Relapses were detected based on self-report and monthly urine testing, which may underestimate actual relapse events. A large sample would also allow subgroup analysis based on those who had relapsed and those who did not, thereby providing further insight into the effects of MMT. Finally, the present study assessed patients in a 12-month window, which may be insufficient for assessing the therapeutic potential of MMT.

Despite these limitations, the present study provided evidence that MMT increases inhibitory control function in heroin-dependent patients and suggested that the therapy may initially work by strengthening cognition-based inhibitory control processes, with behavior-based processes modified in the longer term. This may have implications for determining the minimal effective MMT period. MMT should be beneficial for maintaining heroin withdrawal and inhibitory control function recovery after withdrawal. The present study also suggested that the psychological and emotional state of patients may affect the efficacy of MMT. However, the data did not reveal how the duration and the dose of heroin or methadone affect MMT efficacy. The findings highlight the requirement for more a detailed study with larger samples for periods longer than one year.

\section{Acknowledgements}

The authors would like to thank the Baqiao methadone substitution treatment centers in Xi'an (China) for providing invaluable assistance. The authors are grateful to Dr Wan Yi (Department of Statistics, The Fourth Military Medical University, Xi'an, China) for his statistical assistance, and to Dr Wang Defeng and Dr Liu Kai of the Department of Imaging and Interventional Radiology, Chinese University of Hong Kong (Hong Kong, China) for their editorial contribution to the manuscript. The authors also thank Dr Zheng Dandan and Dr Zhou Zhenyu of GE Healthcare MR Research (Beijing, China) for their support and assistance.

\section{Funding}

The present study was supported by grants from the National Natural Science Foundation of China (grant nos. 81371532, 
81201081, 81471648 and 81401393) and the Clinical Research Foundation of Tangdu Hospital (grant no. 2013LCYJ003).

\section{Authors' contributions}

JJY, WL, DSZ, QL, WW and YRW conceived and designed the experiments. JJY, JZ, JJC, YBL, XJY, JRL and XW performed the experiments. JJY, DSZ and QL analyzed the data. JJY wrote the manuscript.

\section{Availability of data and materials}

The datasets from our database are not publicly available, but can be made available from the corresponding author on reasonable request.

\section{Ethics approval and consent to participate}

The study protocol was approved by the Tangdu Hospital Review Board (the Fourth Military Medical University, Xi'an, China). All participants provided written informed consent prior to their inclusion in the study.

\section{Consent for publication}

All participants provided written informed consent prior to entering into the study. In the informed consent, they agreed to use the data for scientific research without revealing their privacy.

\section{Competing interests}

The authors declare that they have no competing interests.

\section{References}

1. Bracken BK, Trksak GH, Penetar DM, Tartarini WL, Maywalt MA, Dorsey CM and Lukas SE: Response inhibition and psychomotor speed during methadone maintenance: Impact of treatment duration, dose, and sleep deprivation. Drug Alcohol Depend 125: 132-139, 2012.

2. Mintzer MZ, Copersino ML and Stitzer ML: Opioid abuse and cognitive performance. Drug Alcohol Depend 78 225-230, 2005

3. Constantinou N, Morgan CJ, Battistella S, O'Ryan D, Davis P and Curran HV: Attentional bias, inhibitory control and acute stress in current and former opiate addicts. Drug Alcohol Depend 109: $220-225,2010$

4. Verdejo A, Toribio I, Orozco C, Puente KL and Pérez-García M: Neuropsychological functioning in methadone maintenance patients versus abstinent heroin abusers. Drug Alcohol Depend 78: 283-288, 2005.

5. Andersen JM, Olaussen CF, Ripel A and Mørland J: Long-term methadone treatment impairs novelty preference in rats both when present and absent in brain tissue. Pharmacol Biochem Behavior 98: 412-416, 2011.

6. Tramullas M, Martínez-Cué C and Hurlé MA: Chronic methadone treatment and repeated withdrawal impair cognition and increase the expression of apoptosis-related proteins in mouse brain. Psychopharmacology (Berl) 193: 107-120, 2007.

7. Lin WC, Chou KH, Chen CC, Huang CC, Chen HL, Lu CH, Li SH, Wang YL, Cheng YF and Lin CP: White matter abnormalities correlating with memory and depression in heroin users under methadone maintenance treatment. Plos One 7: e33809, 2012.

8. Liao DL, Huang CY, Hu S, Fang SC, Wu CS, Chen WT, Lee TS, Chen PC and Li CS: Cognitive control in opioid dependence and methadone maintenance treatment. PloS One 9: e94589, 2014.
9. Gruber SA, Tzilos GK, Silveri MM, Pollack M, Renshaw PF, Kaufman MJ and Yurgelun-Todd DA: Methadone maintenance improves cognitive performance after two months of treatment. Exp Clin Psychopharmacol 14: 157-164, 2006.

10. Nigg JT: On inhibition/disinhibition in developmental psychopathology: Views from cognitive and personality psychology and a working inhibition taxonomy. Psychol Bull 126: 220-246, 2000.

11. Peles E, Schreiber S, Naumovsky Y and Adelson M: Depression in methadone maintenance treatment patients: Rate and risk factors. J Affect Disord 99: 213-220, 2007.

12. Kavanaugh BC: The role of inhibitory control in the hospitalization of children with severe psychiatric disorders. Clin Neuropsychol 30: 369-370, 2016

13. Lin WC, Chou KH, Chen HL, Huang CC, Lu CH, Li SH, Wang YL, Cheng YF, Lin CP and Chen CC: Structural deficits in the emotion circuit and cerebellum are associated with depression, anxiety and cognitive dysfunction in methadone maintenance patients: A voxel-based morphometric study. Psychiatry Res 201: 89-97, 2012.

14. Verdejo-Garcia A, Lawrence AJ and Clark L: Impulsivity as a vulnerability marker for substance-use disorders: Review of findings from high-risk research, problem gamblers and genetic association studies. Neurosci Biobehav Rev 32: 777-810, 2008.

15. Camchong J, Stenger A and Fein G: Resting-state synchrony during early alcohol abstinence can predict subsequent relapse. Cereb Cortex 23: 2086-2099, 2013.

16. Mechelli A, Henson RN, Price CJ and Friston KJ: Comparing event-related and epoch analysis in blocked design fMRI. Neuroimage 18: 806-810, 2003.

17. Friston KJ, Fletcher P, Josephs O, Holmes A, Rugg MD and Turner R: Event-related fMRI: Characterizing differential responses. Neuroimage 7: 30-40, 1998.

18. Fu LP, Bi GH, Zou ZT, Wang Y, Ye EM, Ma L, Ming-Fan and Yang Z: Impaired response inhibition function in abstinent heroin dependents: An fMRI study. Neurosci Lett 438: 322-326, 2008.

19. Garavan H, Ross TJ, Murphy K, Roche RA and Stein EA: Dissociable executive functions in the dynamic control of behavior: Inhibition, error detection, and correction. Neuroimage 17: 1820-1829, 2002.

20. Menon V, Adleman NE, White CD, Glover GH and Reiss AL: Error-related brain activation during a Go/NoGo response inhibition task. Hum Brain Mapp 12: 131-143, 2001.

21. Swick D, Ashley V and Turken U: Are the neural correlates of stopping and not going identical? Quantitative meta-analysis of two response inhibition tasks. Neuroimage 56: 1655-1665, 2011.

22. Eagle DM, Bari A and Robbins TW: The neuropsychopharmacology of action inhibition: Cross-species translation of the stop-signal and go/no-go tasks. Psychopharmacology (Berl) 199: 439-456, 2008.

23. Kaufman JN, Ross TJ, Stein EA and Garavan H: Cingulate hypoactivity in cocaine users during a GO-NOGO task as revealed by event-related functional magnetic resonance imaging. J Neurosci 23: 7839-7843, 2003.

24. Simmonds DJ, Pekar JJ and Mostofsky SH: Meta-analysis of Go/No-go tasks demonstrating that fMRI activation associated with response inhibition is task-dependent. Neuropsychologia 46: 224-232, 2008

25. Hamilton M: The assessment of anxiety states by rating. Br J Med Psychol 32: 50-55, 1959

26. Clinical practice guideline: Diagnosis and evaluation of the child with attention-deficit/hyperactivity disorder. American Academy of Pediatrics. Pediatrics 105: 1158-1170, 2000.

27. ZW H: The preliminary establishment of opioid protracted withdrawal symptoms self evaluation scale. Chin Mental Health J 17: 294, 2003 (In Chinese).

28. Patton JH, Stanford MS and Barratt ES: Factor structure of the Barratt impulsiveness scale. J Clin Psychol 51: 768-774, 1995.

29. Association AP: Diagnostic and statistical manual of mental disorders, 4th ed, text rev. Washington, DC. 2002.

30. Rubia K, Russell T, Overmeyer S, Brammer MJ, Bullmore ET, Sharma T, Simmons A, Williams SC, Giampietro V, Andrew CM and Taylor E: Mapping motor inhibition: Conjunctive brain activations across different versions of go/no-go and stop tasks. Neuroimage 13: 250-261, 2001.

31. Wang W, Li Q, Wang Y, Tian J, Yang W, Li W, Qin W, Yuan K and Liu J: Brain fMRI and craving response to heroin-related cues in patients on methadone maintenance treatment. Am J Drug Alcohol Abuse 37: 123-130, 2011. 
32. Salo R, Nordahl TE, Galloway GP, Moore CD, Waters C and Leamon MH: Drug abstinence and cognitive control in methamphetamine-dependent individuals. J Subst Abuse Treat 37: 292-297, 2009

33. Schweinsburg AD, Schweinsburg BC, Medina KL, McQueeny T, Brown SA and Tapert SF: The influence of recency of use on fMRI response during spatial working memory in adolescent marijuana users. J Psychoact Drugs 42: 401-412, 2010.

34. Li CS, Luo X, Sinha R, Rounsaville BJ, Carroll KM, Malison RT, Ding YS, Zhang S and Ide JS: Increased error-related thalamic activity during early compared to late cocaine abstinence. Drug Alcohol Depend 109: 181-189, 2010.

35. Harnishfeger KK: The development of cognitive inhibition, In: Interferenee and Inhibition in Cognition. Dempster FN and Brainerd CJ (eds). Academic Press, San Diego, CA, 1995.

36. Bari A and Robbins TW: Inhibition and impulsivity: Behavioral and neural basis of response control. Prog Neurobiol 108: 44-79, 2013.

37. Nambu A, Tokuno $\mathrm{H}$ and Takada M: Functional significance of the cortico-subthalamo-pallidal 'hyperdirect' pathway. Neurosci Res 43: 111-117, 2002.

38. Aron AR and Poldrack RA: Cortical and subcortical contributions to Stop signal response inhibition: Role of the subthalamic nucleus. J Neurosci 26: 2424-2433, 2006.

39. Greenhouse I, Gould S, Houser M, Hicks G, Gross J and Aron AR Stimulation at dorsal and ventral electrode contacts targeted at the subthalamic nucleus has different effects on motor and emotion functions in Parkinson's disease. Neuropsychologia 49: 528-534, 2011

40. Boehler CN, Appelbaum LG, Krebs RM, Hopf JM and Woldorff MG: Pinning down response inhibition in the brain-conjunction analyses of the Stop-signal task. NeuroImage 52: 1621-1632, 2010.
41. Alexander GE, Crutcher MD and DeLong MR: Basal ganglia-thalamocortical circuits: Parallel substrates for motor, oculomotor, 'prefrontal' and 'limbic' functions. Prog Brain Res 85: 119-146, 1990

42. Ghahremani DG, Lee B, Robertson CL, Tabibnia G, Morgan AT, De Shetler N, Brown AK, Monterosso JR, Aron AR, Mandelkern MA, et al: Striatal dopamine $\mathrm{D}(2) / \mathrm{D}(3)$ receptors mediate response inhibition and related activity in frontostriatal neural circuitry in humans. J Neurosci 32: 7316-7324, 2012.

43. Jahfari S, Waldorp L, van den Wildenberg WP, Scholte HS, Ridderinkhof KR and Forstmann BU: Effective connectivity reveals important roles for both the hyperdirect (fronto-subthalamic) and the indirect (fronto-striatal-pallidal) fronto-basal ganglia pathways during response inhibition. J Neurosci 31: 6891-6899, 2011.

44. Li CS, Yan P, Sinha R and Lee TW: Subcortical processes of motor response inhibition during a stop signal task. Neuroimage 41: 1352-1363, 2008

45. Swick D, Ashley V and Turken AU: Left inferior frontal gyrus is critical for response inhibition. BMC Neurosci 9: 102, 2008.

46. Dosenbach NU, Visscher KM, Palmer ED, Miezin FM, Wenger KK, Kang HC, Burgund ED, Grimes AL, Schlaggar BL and Petersen SE: A core system for the implementation of task sets. Neuron 50: 799-812, 2006.

47. Brunia $\mathrm{CH}$ : Waiting in readiness: Gating in attention and motor preparation. Psychophysiology 30: 327-339, 1993.

48. Aron AR, Behrens TE, Smith S, Frank MJ and Poldrack RA: Triangulating a cognitive control network using diffusion-weighted magnetic resonance imaging (MRI) and functional MRI. J Neurosci 27: 3743-3752, 2007. 